\title{
Exploratory Factor Analysis of the Diabetes Self-Efficacy Scale Among Type 2 Diabetes Mellitus in Thailand
}

\author{
Monthida Sangruangake ${ }^{1, *}$, Solikhah Solikhah ${ }^{2}$, Chananya Jirapornkul ${ }^{3}$, and Cameron Hurst ${ }^{4}$ \\ ${ }^{1}$ Doctoral of Public Health Program, KhonKaen University, Thailand \\ ${ }^{2}$ Faculty of Public Health, Universitas Ahmad Dahlan, Indonesia \\ ${ }^{3}$ Departments of Epidemiology and Biostatistics, Faculty of Public Health, KhonKaen University, KhonKaen, Thailand \\ ${ }^{4}$ Center of Excellence in Biostatistics, Faculty of Medicine, Chulalongkorn University, Bangkok, Thailand
}

\begin{abstract}
The aims of this study were to test the construct validity of the Diabetes Management Self-Efficacy Scale (DMSES) among Thai patients with Type 2 Diabetes Mellitus. This methodological study consisted of two stages: (1) Search for existing literature regarding (DMSES) in English to be translated into Thai and further examined for face validity; and (2) explore the factor structure of the resultant instrument and examine its reliability. A selfadministered questionnaire was used to collect the data in Thai T2DM patients in February 2016. The finding showed that the Thai Diabetes Management Self-Efficacy scale (T-DMSES) contained four domains with 20 items on self-efficacy: (1) Diet; (2) Monitor; (3) Physical; and (4) Regimen. The scales based on all retained items showed a high degree of internal consistency (Cronbach's $\alpha=0.89$ ). The conclusions of this study revealed that the T-DMSES contributed to good construct validity. The said questionnaire could be utilized to explore selfefficacy in Thai T2DM patients, as well as being able to develop and evaluate suitable educational interventions to enhance self-efficacy. Next study should emphasis on further validating the T-DMSES, including assessing construct and criterion-based validity, or examining the psychometric properties by utilizing the Confirmatory Factor Analysis.
\end{abstract}

Keywords: Diabetes Self-Efficacy Scale, Exploratory Factor Analysis, Type 2 Diabetes Mellitus.

\section{INTRODUCTION}

Diabetes is considered one of key global public health issues. The estimated number of people with diabetes worldwide was 285 million cases in 2010 and is projected to reach 439 million cases in 2030, the majority of which (69\%) are found in developing countries. ${ }^{1}$ The number of patients with diabetes in Asia was estimated at 113 million cases in 2010 and will reach 180 million cases in $2030 .^{2}$ In a low and middle-income country like Thailand, diabetes represents one of the leading causes of morbidity and mortality. ${ }^{3}$ Currently, there are 3.2 million patients with diabetes in Thailand, and this is estimated to rise by 1.1 million patients in $2035 .^{4}$

Diabetes mellitus type 2 (T2DM) is a disease where patients self-care can substantially reduce the risk, or delay the onset, of T2DM complication. The following tasks can substantially make the improvement of diabetes: Taking medications as prescribed; monitoring diet and blood glucose levels; performing physical activities, and caring for feet. ${ }^{5}$ All of these tasks come as a package after being diagnosed with diabetes. Such

${ }^{*}$ Author to whom correspondence should be addressed. activities needed to be incorporated into daily living and created as behaviors so that patients could control the disease and slow progression and reduce complications. ${ }^{6}$ The process as mentioned above is defined as Diabetes Self-Management (DSM).

The Diabetes Self-Management (DSM) provides support for informed decision-making, self-care behaviors, problem-solving, and active collaboration with the health care team, resulting in improved clinical outcomes, health status, and quality of life. ${ }^{7}$ The management relies on the long-term cooperation of patients in obtaining regular medical care and adhering to treatment plans. $^{8-10}$

Self-efficacy is referred to as an individual's perceptions or beliefs in their capabilities to carry out certain activities. ${ }^{11}$ It is influential to their thinking, feeling, motivation, and behaviors. The person, whose high self-efficacy chooses to perform more challenging tasks, they set higher goals for themselves and adhere to the goals. To take action, individuals who have high selfefficacy exert more effort with more persistence than those who have low self-efficacy. In a case of setbacks, they achieve quicker recovery and continue the commitment to achieving their goals. This efficacious outlook contributes to personal accomplishments, stress reduction, as well as mitigates vulnerability to depression. ${ }^{12}$ 
The Diabetes Management Self-Efficacy Scale (DMSES) was originally developed for use in western populations and assesses the confidence of diabetes patients in their ability to manage their diet, blood sugar, and physical exercise. ${ }^{13}$ This instrument is primarily utilized to measure self-efficacy. From the past to present, the tool has been widely recognized, adapted, and translated to be correctly used in several areas with the different culture, namely, Netherlands, ${ }^{13}$ the United Kingdom, ${ }^{14}$ Australia, ${ }^{9}$ Turkey, ${ }^{15}$ Taiwan, ${ }^{16}$ and Korea. ${ }^{13}$ However, all related researchers provided different findings possibly because of the various characteristics in each area and sample size. As a result, the original version of the instrument is varied from those in other areas as evidenced by a different number of factors to be generated. ${ }^{15,16}$ Although reported as validated, the methodological approaches used in the validation studies were often valued inappropriately. An instrument developed and validated on Western may not be assumed to be valid in Asian populations. Therefore, to apply the instrument to patients with T2DM in Thailand, it is quite necessary to confirm whether it is proper to use the instrument in Thailand. Moreover, based on the literature review, it was found that the development of DMSE instrument has not been validated. The diabetes management self-efficacy scale (DMSES) among Thai population is seen as an important initial step to achieve the goal of preventive services for patients with T2DM. Therefore, the objective of this study is of test reliability and validity of the Thai Diabetes Management Self-Efficacy scale for measurement in Thailand's context.

\section{MATERIALS AND METHOD}

This methodological study consisted of two stages:

(1) Search for existing literature regarding Diabetes Management Self-Efficacy Scale (DMSES) in English to be translated into Thai and further examined for face validity; and

(2) Explore the factor structure of the resultant instrument and examine its reliability.

\section{Phase 1. Instrument translation and face validity}

The Diabetes Management Self-Efficacy Scale (DMSES) was originally a self-administered scale containing 20 items distributed across four factors, each of which had a 5-point scale. ${ }^{13}$ The DMSES items were translated from English into Thai by a forward and backward translation technique. A forward and backward translation technique was performed by two forward and two different backward native bilinguals. ${ }^{17}$ Every translator was not accustomed to the content and had no clinical background. The two bilingual translators, whose native language was Thai, independently proceeded with the forward translation from the original version, and then finalized the most accurate and comprehensible terms by consensus. After that, a bilingual translator, who had not been exposed to the original version and the concepts being examined in the questionnaire, translated the items back to English. After the last two bilingual translators had completed back-translation into the English version, two native English translators compared between the English backtranslated version and the original English version. Any evident discrepancies between the two translated versions were modified. Then, the back-translation process was carried on until the examiner was satisfied with the language equivalency. The field test for the Thai Diabetes Management Self-Efficacy Scale (T-DMSES) was conducted in $20 \mathrm{~T} 2 \mathrm{DM}$ patients to assess the translation quality and the practicality of the test administration. Respondents were asked to read or listen to each item to ensure their understanding of each item.

Phase 2. Evaluating the psychometric properties of the T-DMSES

\section{Sample and study design}

The study was a cross-sectional study design aiming to test the reliability and validity of the T-DMSES in Thai T2DM patients. $700 \mathrm{~T} 2 \mathrm{DM}$ patients were living in both rural and urban areas from The Central and Northeastern Thailand. The T2DM samples were recruited from outpatient diabetes clinics of both community hospitals and University hospitals in KhonKaen province and Bangkok, Thailand. The development of stratification was based on locality, while the determination of sample size was of this study based on factor analysis to establish construct validity: $100=$ fair, $200=$ good, $500=$ very good, and $>1000=$ excellent. ${ }^{18}$ The questionnaire was administered in February-June 2016 to T2DM outpatients aged $\geq 20$ years old who were diagnosed with T2DM for more than three years, able to read or understand Thai language and willing to participate in the study. The authorized person of each hospital gave permission to collect the data, and all participants provided informed consent. Then, the data of the participants were collected until the data collection was completed using the participants' self-report. The study protocol was approved by the ethics committee of KhonKaen University (HE581479), Institutional Review Board at Faculty of Medicine, Chulalongkorn University (IRB035/59), and Bangkok Metropolitan Administration Ethics Committee for Human Research (U005q/59).

2. Demographic and clinical characteristic of DMSES

The original Version of the Diabetes Management SelfEfficacy Scale (DMSES) was a self-administered scale composed of 20 items designed to investigate behaviors in terms of four factors as follows:

(1) specific nutrition and weight, with a subscale of five items designed to determine the stick and adjust to diet when away from home, stick to diet on vacation and party, control weight;

(2) general nutrition and medical treatment, with a subscale of nine items designed to determine the choose and variation in nutrition, stick to diet most of the time, adjust diet when ill and stress check feet, consult physician for diabetes control, used of medication;

(3) physical exercise, with a subscale of three items designed to determine the extra and take care of training on physician's advice; and

(4) blood sugar, with a subscale of three items designed to determine the remedy low and high and self-control blood sugar.

The items were scored on a 5-point Likert-type scale, with higher scores indicating higher self-efficacy. From the past to present, the instrument has been widely recognized, adapted, and translated to be properly used in several areas with a different culture. ${ }^{9,13,15,16}$

\section{Statistical analysis}

Demographic data of the participants in this study were summarized using descriptive statistics. Exploratory factor analysis (EFA) was a method to confirm the construct validity of instrument model to seek a single or set of predefined constructs. EFA 
Table I. Patient characteristics.

\begin{tabular}{|c|c|}
\hline Characteristics $(n=700)$ & $n(\%)$ \\
\hline \multicolumn{2}{|l|}{ Parts of Hospital } \\
\hline Phuphaman Hospital & $60(8.57)$ \\
\hline Srinagarind Hospital & $78(11.14)$ \\
\hline Wechkaroonrasm Hospital & $242(34.57)$ \\
\hline Chulalongkorn Hospital & $320(45.71)$ \\
\hline \multicolumn{2}{|l|}{ Gender } \\
\hline Male & $208(29.71)$ \\
\hline Female & $492(70.29)$ \\
\hline \multicolumn{2}{|l|}{ Age (Years) } \\
\hline Mean (SD) & $65.16(10.94)$ \\
\hline Range & $26-95$ \\
\hline \multicolumn{2}{|l|}{ Marital Status } \\
\hline Single & $57(8.14)$ \\
\hline Married & $465(66.43)$ \\
\hline Divorce & $165(23.57)$ \\
\hline Separate & $13(1.86)$ \\
\hline \multicolumn{2}{|l|}{ Education } \\
\hline No formal education & $47(6.71)$ \\
\hline Elementary school & $381(54.43)$ \\
\hline High school & $146(20.86)$ \\
\hline Bachelor degree & $99(14.14)$ \\
\hline Master degree & $25(3.57)$ \\
\hline Higher degree & $2(0.29)$ \\
\hline \multicolumn{2}{|l|}{ Religion } \\
\hline Buddhism & $543(77.57)$ \\
\hline Islam & $152(21.71)$ \\
\hline Christianity & $5(0.71)$ \\
\hline \multicolumn{2}{|l|}{ Monthly income } \\
\hline$<4,999$ baht (Less than 143 USD) & $318(45.43)$ \\
\hline $5,000-9,999$ baht (143 to 287 USD) & $95(13.56)$ \\
\hline $10,000-14,999$ baht ( 287 to 413 USD) & $86(12.29)$ \\
\hline $15,000-19,999$ baht (413 to 575 USD) & $48(6.86)$ \\
\hline $20,000-24,999$ baht ( 575 to 718 USD) & $48(6.86)$ \\
\hline$>25,000$ baht (more than 718 USD) & $105(15.00)$ \\
\hline \multicolumn{2}{|l|}{ BMI } \\
\hline$<18.5$ & $20(2.86)$ \\
\hline $18.5-24.9$ & $279(39.86)$ \\
\hline 25-29.9 & $219(31.29)$ \\
\hline$\geq 30$ & $182(26)$ \\
\hline \multicolumn{2}{|l|}{$($ Mean $=27.08 ; S D=6.32)$} \\
\hline \multicolumn{2}{|l|}{ Duration of diabetes } \\
\hline$\leq 5$ years & $85(12.14)$ \\
\hline$>5$ years & $615(87.86)$ \\
\hline \multicolumn{2}{|l|}{$($ Mean $=13.53 ; \mathrm{SD}=8.34)$} \\
\hline \multicolumn{2}{|l|}{ Family history of DM } \\
\hline Yes & $370(52.86)$ \\
\hline No & $330(47.14)$ \\
\hline \multicolumn{2}{|l|}{ Co-morbidity } \\
\hline Hyperlipidemia & $605(86.43)$ \\
\hline Hypertension & $603(86.14)$ \\
\hline Heart disease & $38(5.43)$ \\
\hline Renal disease & $188(26.86)$ \\
\hline Treatment of DM & \\
\hline No drug & $14(2)$ \\
\hline Oral hyperglycemic agent $(\mathrm{OHA})$ & $408(58.29)$ \\
\hline Insulin sensitizer & $94(13.43)$ \\
\hline Both $\mathrm{OHA}$ and insulin sensitivity & $184(26.29)$ \\
\hline Smoking & \\
\hline No & $589(84.14)$ \\
\hline Previously & $88(12.57)$ \\
\hline Yes & $23(3.29)$ \\
\hline Alcohol & \\
\hline No & 569 (81.29) \\
\hline Previously & $89(12.71)$ \\
\hline Yes & $42(6)$ \\
\hline Hba1c & \\
\hline$\leq 7 \%$ & $317(45.29)$ \\
\hline$>7 \%$ & $383(54.71)$ \\
\hline$($ Mean $=7.58 ; S D=1.70)$ & \\
\hline
\end{tabular}

was used to initially determine the number of latent factors or the pattern of correlations between the common factors and the indicators. Parallel analysis based on Principal Components Analysis was used to determine the nature of the underlying factors. An Oblique (Promax) model was adopted after principal axis factoring models were compared with orthogonal (Varimax) and oblique rotations. Eigenvalues greater than 1 and factor loading cut-off of less than 0.2 were used to acquire the best fitting structure and the accurate number of factors. The $\mathrm{R}$ statistics package by v 2.3.0; R CoreTeam, 2015 was used to conduct all analyses, and the R library lavaan was utilized to proceed with the complete factor analysis. ${ }^{19}$ Cronbach's alpha was used to evaluate the internal consistency reliability of the overall instrument and the individual subscales. Acceptable reliability for the scale and all subscales was designated to be $\alpha>0.7 .^{20}$

\section{RESULTS AND DISCUSSION}

\subsection{Demographic Characteristics of Participants in a Validation Study}

There were totally 700 T2DM patients completing the questionnaire (Response rate of $94.3 \%$ ), with age ranging from 26 to 95 years old $($ Mean $=65.16, \mathrm{SD}=10.94)$. The research participants consisted of $29.71 \%$ males and $70.29 \%$ females. They had been diagnosed with diabetes for 13.53 years on average $(\mathrm{SD}=$ 8.34). Other demographic characteristics of the participants were presented in Table I.

\subsection{Exploratory Factor Analysis (EFA)}

According to the exploratory factor analysis in 700 patients, a parallel group study was conducted, and four-factor structure was deemed appropriate (Fig. 1). The KMO was 0.88 and Bartlett's sphericity test was significant $\left(\chi^{2}=8576.884\right.$, df $=190, p<$ 0.001 ) indicating reasonable adequacy of the data for factor analysis. The measurement model for Thai diabetes management selfefficacy scale (T-DMSES) and 20 items was conducted as shown in Figure 2. Nevertheless, the authors decided to retain two of the 20 lower loading items since their inclusion was quite strongly supported by the literature. The principal axis factoring with an oblique rotation was subsequently utilized to explore the factor structure, and the resultant loadings are giving in Table II.

\subsection{Internal Consistency}

Based on the four factor model, the reliability of the TDMSES was evaluated for internal consistency (Cronbach's

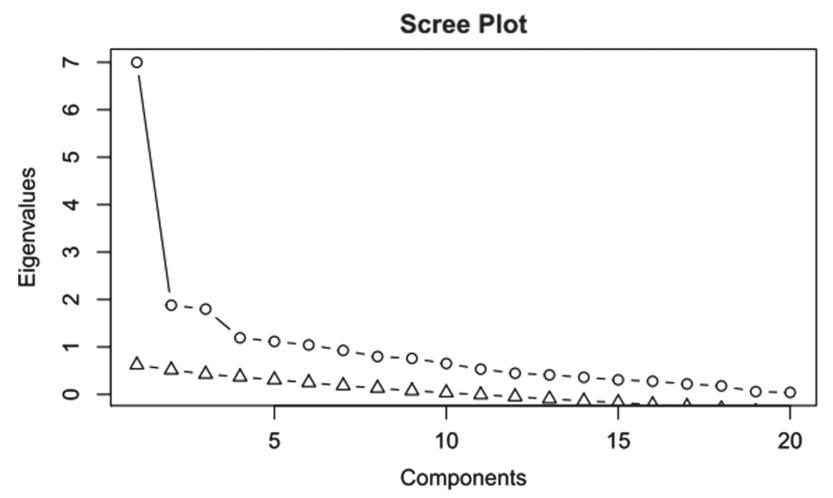

Fig. 1. Scree plot for determination of number of factors. 


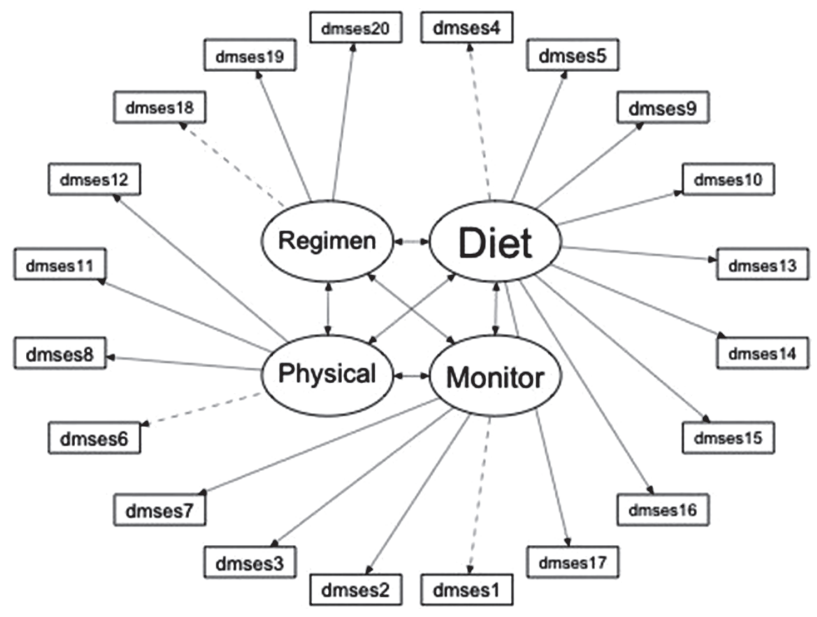

Fig. 2. Measurement model for Thai diabetes management self-efficacy scale (T-DMSES).

alpha). The alpha value of 0.89 indicates sufficiently high reliability to provide confidence in interpreting the score.

\section{DISCUSSION}

To the best of the authors' knowledge, this is the first time that a version of the DMSES has been tested for content validity in Thai T2DM patients. Our instrument provided strong internal consistency reliability. Moreover, it was found that most of the items strongly aligned with their hypothesized constructs, and enabled researchers and practitioners to gain a better understanding of selfefficacy for seeking interventions. Based on the findings of our study, the T-DMSES revealed that relevant underlying domains for the evaluation of self-efficacy included Diet, Monitor, Physical, and Regimen. Some domains of the T-DMSES were consistent with those identified in previous studies. ${ }^{16}$ To conduct the exploratory factor analysis, the authors chose the method of principal axis factoring with oblique rotation to extract factors. The results of such analysis suggested that there were 2 items from an original version of measurement with factor loading $<0.2$, but they were likely to be associated with self-efficacy in Thai T2DM patients. Therefore, we decided to force two of these 20 items into the model, and see if they should still be excluded in a subsequent confirmatory factor analysis. In particular, blood glucose levels could be checked by myself if necessary, as well as being able to increase blood glucose levels when glucose levels in my blood are too low. Our research had some limitations. First, we considered recruiting T2DM patients from only the Central and Northeastern part of Thailand. The representativeness of this sample for all Thai T2DM patients was not possible. Second, regarding the validation process, T-DMSES had not yet undergone a construct or criterion-based validation at this stage. Even now, there was no evidence that this instrument could either discriminate between T2DM patients who achieved blood sugar levels and those who could not achieve blood sugar levels, or be used to evaluate the efficiency of a program to enhance self-management (predictive validity). Nonetheless, this study also had some major strength. First, our validation of T-DMSES is the most comprehensive

Table II. Factor loading of the T-DMSES from principal axis factoring.

\begin{tabular}{|c|c|c|c|c|c|}
\hline Factors & Items & Diet & Monitor & Physical & Regimen \\
\hline \multicolumn{6}{|l|}{ Diet (9 item) } \\
\hline dmses 4 & I can choose to eat good and healthy foods that are beneficial to my health & 0.77 & - & - & - \\
\hline dmses5 & I can choose to eat various foods to maintain a healthy diet plan & 0.79 & - & - & - \\
\hline dmses9 & I can maintain a healthy diet plan in the event that I get sick & 0.26 & - & - & - \\
\hline dmses 10 & I can follow a healthy diet plan regularly & 0.70 & - & - & - \\
\hline dmses13 & I can follow a healthy diet plan even when I am not at home & 0.88 & - & - & - \\
\hline dmses 14 & $\begin{array}{l}\text { I can choose from various foods to maintain a healthy diet plan when I am not at } \\
\text { home }\end{array}$ & 0.95 & - & - & - \\
\hline dmses 15 & I can follow a healthy diet plan during festivals, traditions, or rituals & 0.93 & - & - & - \\
\hline dmses16 & $\begin{array}{l}\text { I can choose to eat various foods to maintain a healthy diet plan when I eat foods at } \\
\text { parties }\end{array}$ & 0.94 & - & - & - \\
\hline dmses 17 & I can maintain a healthy diet plan when I am feeling stressed or worried & 0.31 & - & - & - \\
\hline \multicolumn{6}{|c|}{ Monitor (4 Items) } \\
\hline dmses1 & I can check blood glucose levels by myself if necessary & - & * & - & - \\
\hline dmses2 & $\begin{array}{l}\text { I can reduce blood glucose levels when glucose levels in my blood are too high (for } \\
\text { example, changing the kinds of foods I eat). }\end{array}$ & - & 0.36 & - & - \\
\hline dmses3 & $\begin{array}{l}\text { I can increase blood glucose levels when glucose levels in my blood are too low (for } \\
\text { example, changing the kinds of foods I eat) }\end{array}$ & - & * & - & - \\
\hline dmses7 & $\begin{array}{l}\text { I can attend to my feet (for example, cutting toe nails and taking care of myself not } \\
\text { causing wounds). }\end{array}$ & - & 0.21 & - & - \\
\hline \multicolumn{6}{|c|}{ - } \\
\hline dmses6 & I can control my body weight and maintain appropriate weight ranges & - & - & 0.28 & - \\
\hline dmses8 & $\begin{array}{l}\text { I can exercise and perform sufficient physical activity (for example, walking, aerobic } \\
\text { dancing, muscle exercise, etc.) }\end{array}$ & - & - & 0.67 & - \\
\hline dmses11 & I can increase the amount that I exercise if a doctor advises me to do so & - & - & 0.88 & - \\
\hline dmses 12 & In the case that I exercise more, I can modify my healthy diet plan & - & - & 0.82 & - \\
\hline \multicolumn{6}{|c|}{ Regimen (3 Items) } \\
\hline dmses 18 & $\begin{array}{l}\text { I can schedule an appointment to see a doctor four times a year to check my } \\
\text { diabetes }\end{array}$ & - & - & - & 0.43 \\
\hline dmses19 & I can take medicines as prescribed by a doctor & - & - & - & 0.89 \\
\hline dmses20 & I can keep taking medicines continuously when I am sick & - & - & - & 0.70 \\
\hline
\end{tabular}

Note: * Low loading items forced into model. 
evaluation of psychometric properties of the DMSES instrument ever conducted in any population. Our study was multi-center and captured the full spectrum of healthcare available in Thailand. In contrast, all previous studies attempting to validate DMSES have used samples of between 88 to 440 patients invariably collected from a single site. Our study applied an appropriate exploratory factor analysis, i.e., principal axis factoring, to identifying the domains underlying the T-DMSES, and we planned to conduct Confirmatory Factor Analysis and criterion-based validation in our next study. On the contrary, the most commonly used instrument for evaluating diabetes management self-efficacy, DMSES, ${ }^{13}$ was structured in such a way as to cause difficulties in full psychometric validation.

\section{CONCLUSION}

The T-DMSES contributed to good psychometric regarding construct validity and internal consistency reliability, suggesting its utility in evaluating self-efficacy in Thai T2DM patients. In the future, T-DMSES would be likely to provide valuable insights into the epidemiology of diabetes management self-efficacy, and may also be used to assess interventions to reduce ineffective self-care in T2DM patients. In turn, this would potentially reduce the incidence and mortality of patients with type 2 diabetes mellitus complications.

\section{Conflict of Interests}

The authors declare that there is no conflict of interests in publishing this paper.

Acknowledgments: The authors would like to express sincere thanks to Van Der Bijl for permission of using the original version of DMSE, all T2DM patients for participation in this study, and all research assistants for assistance in the data collection in Khon Kaen province and Bangkok, Thailand.

\section{References and Notes}

1. J. E. Shaw, R. A. Sicree, and P. Z. Zimmet, Diabetes Res. Clin. Pract. 87, 1 (2010).

2. J. C. N. Chan, V. Malik, W. Jia, T. K. Adowaki, C. S. Yajnik, K. H. Yoon, and F. B. Hu, JAMA 301, 20 (2009).

3. Y. Porapakkham, C. Rao, and J. Pattaraarchachai, Population Health Metrics 4, 14 (2010).

4. D. Whiting, L. Guariguata, C. Weil, and J. Shaw, Diabetes Research and Clinical Practice 94, 3 (2011).

5. American Diabetes Association, Diabetes Care 27 (Suppl. 1) (2004).

6. Y. Xu, D. Toobert, C. Savage, W. Pan, and K. Whitmer, Research in Nursing and Health 31, 6 (2008).

7. M. M. Funnell, T. L. Brown, B. P. Childs, L. B. Haas, G. M. Hosey, B. Jensen, M. Maryniuk, M. Peyrot, J. D. Piette, D. Reader, L. M. Siminerio, K. Weinger, and M. A. Weiss, Diabetes Care 35 (Suppl. 1) (2012).

8. K. R. Lorig, D. S. Sobel, A. L. Stewart, B. W. Brown, A. Bandura, P. Ritter, V. M. Gonzalez, D. D. Laurent, and H. R. Holman, Med. Care 37, 1 (1999).

9. J. McDowell, M. Courtney, H. Edwards, and L. Shortridge-Baggett, International Journal of Nursing Practice 11, 4 (2005).

10. D. J. Toobert, S. E. Hampson, and R. E. Glasgow, Diabetes Care 23, 7 (2000).

11. A. Bandura, Psychological Review 84, 2 (1977).

12. A. Bandura, Health Education and Behavior 31, 2 (2004).

13. E. H. Lee, J. J. van der Bijl, L. M. Shortridge-Baggett, S. J. Han, and S. H. Seung Hei Moon, International Journal of Endocrinology (2015).

14. J. Sturt, H. Hearnshaw, and M. Wakelin, Primary Health Care Research and Development 11 (2010).

15. M. Kara, J. J. van der Bijl, L. M. Shortridge-Baggett, T. Asti, and S. Erguney, International Journal of Nursing Studies 43, 5 (2006)

16. S.-F. Vivienne Wu, M. Courtney, H. Edwards, J. McDowell, L. M. ShortridgeBaggett, and P.-J. Chang, International Journal of Nursing Studies 45, 4 (2008).

17. R. W. Brislin, Journal of Cross Cultural Psychology 1, 3 (1970).

18. A. L. Comrey and H. Lee, Lawrence Erlbaum Associates, Publishers (1992).

19. Y. Rosseel, Journal of Statistical Software 48, 2 (2012).

20. The Handbook of Psychological Testing, 2nd edn., Routledge, London (2000). 